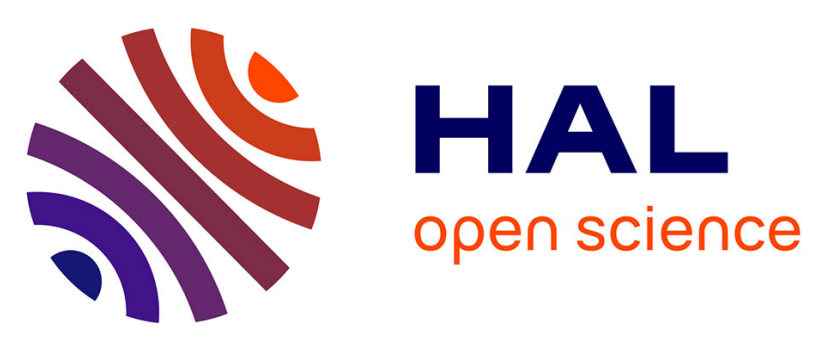

\title{
Convergence of the Upwind Interface Source method for hyperbolic conservation laws
}

Benoît Perthame, Chiara Simeoni

\section{To cite this version:}

Benoit Perthame, Chiara Simeoni. Convergence of the Upwind Interface Source method for hyperbolic conservation laws. Proceedings of the Ninth International Conference on Hyperbolic Problems, Mar 2002, CalTech, Pasadena, United States. pp.61-78, 10.1007/978-3-642-55711-8_5 . hal-00922680v2

\section{HAL Id: hal-00922680 \\ https://hal.science/hal-00922680v2}

Submitted on 30 Dec 2013

HAL is a multi-disciplinary open access archive for the deposit and dissemination of scientific research documents, whether they are published or not. The documents may come from teaching and research institutions in France or abroad, or from public or private research centers.
L'archive ouverte pluridisciplinaire HAL, est destinée au dépôt et à la diffusion de documents scientifiques de niveau recherche, publiés ou non, émanant des établissements d'enseignement et de recherche français ou étrangers, des laboratoires publics ou privés. 


\title{
Convergence of the Upwind Interface Source method for hyperbolic conservation laws
}

\author{
Benoît Perthame and Chiara Simeoni \\ Département de Mathématiques et Applications - École Normale Supérieure \\ 45, rue d'Ulm - 75230 Paris Cedex 05 - France \\ Benoit.Perthame@ens.fr, Chiara.Simeoni@ens.fr
}

Summary. This paper deals with typical questions arising in the analysis of numerical approximations for scalar conservation laws with a source term. We focus our attention on semi-discrete finite volume schemes, in the general case of a nonuniform spatial mesh. To define appropriate discretizations of the source term, we introduce the formalism peculiar to the Upwind Interface Source method and we establish conditions on the numerical functions so that the discrete solver preserves the steady state solutions. Then we formulate a rigorous definition of consistency, adapted to the class of well-balanced schemes, for which we are able to prove a Lax-Wendroff type convergence theorem. Some examples of numerical methods are discussed, in order to validate the arguments we propose.

http://link.springer.com/chapter/10.1007\%2F978-3-642-55711-8_5

\section{Introduction}

We consider a scalar conservation law with a source term, in one space dimension,

$$
\frac{\partial u}{\partial t}+\frac{\partial A(u)}{\partial x}+B(x, u)=0, \quad t \in \mathbb{R}_{+}, x \in \mathbb{R}
$$

with $u(t, x) \in \mathbb{R}$ and a real-valued flux function $A$, associated with a Cauchy problem by introducing the initial condition

$$
u(0, x)=u^{0}(x) \in L^{1}(\mathbb{R}) \cap L^{\infty}(\mathbb{R}) .
$$

We set

$$
a(u)=A^{\prime}(u) \in \mathcal{C}^{1}(\mathbb{R})
$$

and we restrict our analysis to a particular form of source term,

$$
B(x, u)=z^{\prime}(x) b(u), \quad z^{\prime} \in L^{1}(\mathbb{R}), b \in \mathcal{C}^{1}(\mathbb{R}) .
$$

This is suggested by the usual application of hyperbolic conservation laws as simple mathematical models in continuum mechanics: in the Saint-Venant system for shallow waters, for instance, $z(x)$ describes the bottom topography. The equation (1) is endowed with the family of entropy inequalities

$$
\frac{\partial S(u)}{\partial t}+\frac{\partial \eta(u)}{\partial x}+S^{\prime}(u) B(x, u) \leq 0, \quad \eta^{\prime}(u)=S^{\prime}(u) a(u)
$$


for any pair of a convex entropy function $S$ and the corresponding entropy flux $\eta$ (see [21] and [24]). Under stronger assumptions on the source term, Kružkov proved in [21] existence and uniqueness of the entropy solution for the initial value problem (1)-(2), in the functional space $L^{\infty}\left([0, T) ; L^{1}(\mathbb{R})\right)$, for all $T \in \mathbb{R}_{+}$. Many results concerning the convergence of numerical approximations for the entropy solution of hyperbolic conservation laws are inspired by this fundamental theory.

In the case of singular source terms (i.e. the function $z(x)$ is discontinuous), a remarkable uniqueness result has recently been proved by Vasseur [31].

The presence of source terms modifies the analytical properties of the equation (1), in comparison with the homogeneous case. More specifically, a fundamental change is the occurrence of other kinds of steady state solutions, resulting from the balance between source terms and internal forces, given by the formula

$$
D(u)+z(x)=C^{s t}, \quad D^{\prime}(u)=\frac{a(u)}{b(u)} .
$$

This fact also influences the numerical approach to the problem, as it was pointed out by several authors (refer to [15] and [27]), in order to investigate discrete approximations preserving the properties of the continuous system.

A well-known difficulty encountered in the numerical treatment of hyperbolic conservation laws with a source term relates to the approximation of such a source term, to assure that the scheme preserves the steady state solutions at discrete level.

Initially for scalar problems, Greenberg, LeRoux and others introduced the notion of well-balanced schemes (see [15],[16] for details). This definition has been further developed by Gosse and LeRoux [11], which used a reformulation of the source terms by means of non-conservative products to derive numerical fluxes at the interfaces of an unstructured mesh. A recent approach by LeVêque [27] is based on the Godunov scheme extended for an appropriately modified system. Botchorishvili, Perthame and Vasseur present in [2] a kinetic scheme, that maintains steady states and which is proved to converge when stiff source terms are considered. Using interfacial values, instead of the cell-averages, for the source term, Jin proposes in [17] a rather simple method for capturing steady state solutions with a high order accuracy. Previous schemes have also been modified for this target by Bermudez and Vasquez [1] and some different approaches are developed in [22],[23] and [18]. Quite recently, these kinds of numerical processing have been extended to hyperbolic systems of balance laws (like the Saint-Venant system for shallow waters), to obtain stable schemes which preserve the steady states (see [8], $[13],[14]$ and [28], for instance). In particular, one of the main conclusions in [28] is that, while preserving steady states, well-balanced schemes can also enjoy stability under the usual CFL condition (independent of $z^{\prime}$ ).

The aim of this paper is to present a general consistency condition for discrete approximations of equation (1). In fact, to analyze theoretical prop- 
erties of numerical solvers for a conservation law with a source term, the only classical condition on the flux function is not enough and specific definitions for the discrete source term are required.

The outline of the paper is the following. In Section 2, we illustrate the Upwind Interface Source method, which consists in upwinding source terms at the interfaces of the mesh cells, as usual for the fluxes according to the finite volume formalism. Then, in Section 3, we consider discretizations which preserve steady state solutions (well-balanced schemes) and we review several classical methods to build such schemes. The question of consistency is addressed in Section 4 and we show that well-balanced schemes are consistent in the sense we have established. By using these arguments, in Section 5, we finally prove an extension of the Lax-Wendroff theorem.

\section{Upwind Interface Source method}

The finite volume method is possible for treating numerically hyperbolic systems of conservation laws, it is robust and presents the advantage to be conservative (we refer to [6] for a survey of its properties). We look at the semi-discrete scheme, called method of lines, where only a space discretization of equation (1) is performed.

We consider a mesh of $\mathbb{R}$ made up of cells $\mathcal{C}_{i}$, with center $x_{i}, i \in \mathbb{Z}$, and nonuniform length $\Delta x_{i}$. We denote by $x_{i+\frac{1}{2}}$ the cell interfaces, so that the control volume can be identified as $\mathcal{C}_{i}=\left[x_{i-\frac{1}{2}}, x_{i+\frac{1}{2}}\right)$ and $x_{i}=\frac{x_{i-\frac{1}{2}}+x_{i+\frac{1}{2}}}{2}$. Then, we construct a piecewise constant representation of the function $z(x)$ on the mesh, whose coefficients are $z_{i}=\frac{1}{\Delta x_{i}} \int_{\mathcal{C}_{i}} z(x) d x$ for example.

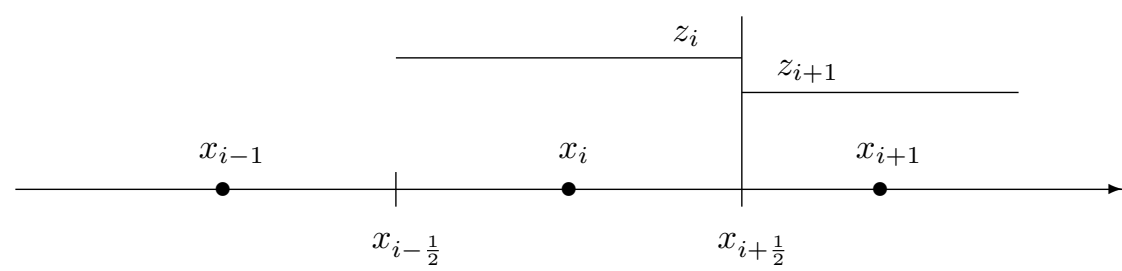

In this context, the discrete unknowns are expected to be approximations of the mean values of $u$ on the mesh cells (the conservative quantities are cell centered),

$$
u_{i}(t) \approx \frac{1}{\Delta x_{i}} \int_{\mathcal{C}_{i}} u(t, x) d x, \quad t \in \mathbb{R}_{+}, i \in \mathbb{Z},
$$

while the numerical fluxes are defined at the interfaces of the mesh.

To correctly treat the source term is more difficult than it seems and centered schemes give unsatisfactory results, as it is well reported in the literature: a direct discretization of the source term by cell-averages, for instance, 
can not preserve the steady state solutions. A better approach is based on the Upwind Interface Source method (upwinding of external terms was originally formulated by Roe [29]), where the source term is also upwinded at the interfaces.

The general finite volume scheme for equation (1) can thus be written in the following explicit form,

$$
\Delta x_{i} \frac{d u_{i}}{d t}+\left(A_{i+\frac{1}{2}}-A_{i-\frac{1}{2}}\right)+\mathcal{B}_{i-\frac{1}{2}}^{+}+\mathcal{B}_{i+\frac{1}{2}}^{-}=0,
$$

dropping the time dependence of the numerical functions for simplicity.

We proceed to explain the notation in the previous formula. We first introduce the discrete fluxes

$$
A_{i+\frac{1}{2}}=\mathcal{A}\left(u_{i}, u_{i+1}\right), \quad \mathcal{A} \in \mathcal{C}^{1},
$$

where the numerical function $\mathcal{A}$ is chosen as a consistent approximation of the analytical flux,

$$
\mathcal{A}(u, u)=A(u) .
$$

Because of the choice of particular source terms (4), the function $z(x)$ is defined up to a constant. Therefore, without loss of generality, we suppose the source term is discretized at the cell interfaces by means of functions

$$
\mathcal{B}_{i+\frac{1}{2}}^{ \pm}=\mathcal{B}^{ \pm}\left(u_{i}, u_{i+1}, z_{i+1}-z_{i}\right), \quad \mathcal{B}^{ \pm} \in \mathcal{C}^{2},
$$

and, in view of (4), it is natural to impose

$$
\mathcal{B}^{+}(u, v, 0)=\mathcal{B}^{-}(u, v, 0)=0 .
$$

The last condition refers to the interpretation of the numerical solver (7), applied to the model (1)-(4). According to the finite volume formalism, we can identify

$$
\mathcal{B}_{i-\frac{1}{2}}^{+}+\mathcal{B}_{i+\frac{1}{2}}^{-} \approx \int_{\mathcal{C}_{i}} z^{\prime}(x) b(u) d x
$$

formally, this leads to deduce

$$
\mathcal{B}_{i+\frac{1}{2}}^{-}+\mathcal{B}_{i+\frac{1}{2}}^{+} \approx \int_{x_{i}}^{x_{i+\frac{1}{2}}} z^{\prime}(x) b(u) d x+\int_{x_{i+\frac{1}{2}}}^{x_{i+1}} z^{\prime}(x) b(u) d x,
$$

that is a way to perform an interfacial approximation of zero order terms. Such a discretization is also upwinded, in the sense that $\mathcal{B}_{i+\frac{1}{2}}^{-}$represents the contribution of the waves coming from the left of the interface $x_{i+\frac{1}{2}}$ and moving towards the cell $\mathcal{C}_{i}$ if they have a nonpositive velocity, while $\mathcal{B}_{i+\frac{1}{2}}^{+}$ represents the waves moving forwards from the right of $x_{i+\frac{1}{2}}$ and counted only if they have nonnegative velocity. Notice that, when the problem becomes homogeneous (for example, $z^{\prime}(x)=0$ in equation (4), motivated by 
the analogy with the Saint-Venant model), this scheme reduces to the usual finite volume approximation for a scalar conservation law.

We observe that all what is stated in this section and in the following is also valid for a fully explicit scheme (obtained, for instance, using a standard forward Euler method for the time discretization),

$$
\frac{\Delta x_{i}}{\Delta t}\left(u_{i}^{n+1}-u_{i}^{n}\right)+\left(A_{i+\frac{1}{2}}^{n}-A_{i-\frac{1}{2}}^{n}\right)+\mathcal{B}_{i-\frac{1}{2}}^{n,+}+\mathcal{B}_{i+\frac{1}{2}}^{n,-}=0,
$$

where we introduce a time-step $\Delta t$ and set $t_{n}=n \Delta t, n \in \mathbb{N}$. We then have to consider an additional restriction on the size of the ratio $\frac{\Delta t}{\Delta x_{i}}$, the usual CFL condition, to guarantee numerical stability.

\section{Well-balanced schemes}

We define general conditions on the discretizations $\mathcal{B}_{i+\frac{1}{2}}^{ \pm}$so that the numerical scheme (7) preserves the steady state solutions. Note that all the methods developed in the references mentioned above are compatible with the formalism introduced in Section 2 and can be put in form (7), as we will do later for some particular cases.

By integrating the stationary equation associated with (1)-(4), we obtain the algebraic relation (6) for smooth steady state solutions. A discrete version is given by

$$
D\left(u_{i}\right)+z_{i}=C^{s t}, \quad \forall i \in \mathbb{Z} .
$$

We consider appropriate hypotheses on $D$, to ensure the existence of a unique Lipschitz continuous solution of that problem, namely that $D$ is strictly monotonic. This assumption is restrictive and not always satisfied in realistic situations, but it is usually made (refer to [2], for instance).

For all initial data defined according to (14), a solver preserving steady states has to verify

$$
\left(A_{i+\frac{1}{2}}-A_{i-\frac{1}{2}}\right)+\mathcal{B}_{i-\frac{1}{2}}^{+}+\mathcal{B}_{i+\frac{1}{2}}^{-}=0 .
$$

This last statement can be formulated in terms of numerical functions, thanks to definition (8) and (10), so that it writes

$$
\begin{aligned}
\mathcal{A}\left(u_{i}, u_{i+1}\right)-\mathcal{A}\left(u_{i-1}, u_{i}\right) & +\mathcal{B}^{+}\left(u_{i-1}, u_{i}, z_{i}-z_{i-1}\right) \\
& +\mathcal{B}^{-}\left(u_{i}, u_{i+1}, z_{i+1}-z_{i}\right)=0,
\end{aligned}
$$

for all $u_{i-1}, u_{i}, u_{i+1}$, such that $D\left(u_{j}\right)+z_{j}=H, j=i-1, i, i+1$. In particular, we choose alternatively $u_{i-1}=u_{i}$ and $u_{i}=u_{i+1}$ (then we deduce, respectively, $z_{i-1}=z_{i}$ and $z_{i}=z_{i+1}$ ), to obtain equivalent conditions at the interfaces, also exploiting properties (9) and (11),

$$
\begin{aligned}
\mathcal{A}\left(u_{i}, u_{i+1}\right)-A\left(u_{i}\right)+\mathcal{B}^{-}\left(u_{i}, u_{i+1}, z_{i+1}-z_{i}\right) & =0, \\
A\left(u_{i+1}\right)-\mathcal{A}\left(u_{i}, u_{i+1}\right)+\mathcal{B}^{+}\left(u_{i}, u_{i+1}, z_{i+1}-z_{i}\right) & =0 .
\end{aligned}
$$

We summarize the previous statements in the following proposition. 
Lemma 1. A numerical scheme in form (7)-(11) is well-balanced, i.e. it preserves the steady state solutions (14), if and only if the equalities

$$
\begin{aligned}
& \mathcal{A}(u, v)-A(u)+\mathcal{B}^{-}\left(u, v, z_{+}-z_{-}\right)=0, \\
& A(v)-\mathcal{A}(u, v)+\mathcal{B}^{+}\left(u, v, z_{+}-z_{-}\right)=0,
\end{aligned}
$$

hold true, for all $u, v, z_{-}, z_{+}$such that

$$
D(u)+z_{-}=D(v)+z_{+} .
$$

We call well-balanced or Steady State Preserving schemes the numerical solvers for the problem (1)-(2) which satisfy those conditions.

We present some numerical schemes to which Lemma 1 applies. We check these approaches enable to preserve the discrete steady state solutions, according to the result stated above.

B.P.V. method In [2], the authors introduce their solver in a compact form, taking into account the source term directly in the definition of the numerical fluxes,

$$
\Delta x_{i} \frac{d u_{i}}{d t}+\left(A_{i+\frac{1}{2}}^{-}-A_{i-\frac{1}{2}}^{+}\right)=0,
$$

with

$$
A_{i+\frac{1}{2}}^{-}=\mathcal{A}\left(u_{i}, u_{i+1}^{-}\right), \quad A_{i-\frac{1}{2}}^{+}=\mathcal{A}\left(u_{i-1}^{+}, u_{i}\right) .
$$

The numerical flux used in [2] is given by a standard Engquist-Osher function, but one readily finds out that similar methods can be formulated with any consistent flux function $\mathcal{A}$. The points $u_{i+1}^{-}$and $u_{i-1}^{+}$are defined by means of the relations

$$
\begin{aligned}
& D\left(u_{i+1}^{-}\right)+z_{i}=D\left(u_{i+1}\right)+z_{i+1}, \\
& D\left(u_{i-1}^{+}\right)+z_{i}=D\left(u_{i-1}\right)+z_{i-1} .
\end{aligned}
$$

To reproduce this scheme in form (7), we identify

$$
\mathcal{B}_{i-\frac{1}{2}}^{+}=\mathcal{A}\left(u_{i-1}, u_{i}\right)-\mathcal{A}\left(u_{i-1}^{+}, u_{i}\right), \quad \mathcal{B}_{i+\frac{1}{2}}^{-}=\mathcal{A}\left(u_{i}, u_{i+1}^{-}\right)-\mathcal{A}\left(u_{i}, u_{i+1}\right) .
$$

For a steady state, we immediately deduce from (14), (20) and (21) that

$$
u_{i+1}^{-}=u_{i}, \quad u_{i-1}^{+}=u_{i},
$$

then resulting in the conditions (15) and (16).

This method extends to more general classes of function $D$, such as quadratic functions, and it also applies to hyperbolic systems of conservation laws endowed with a kinetic interpretation (see [28]).

We remark that, combined with specific approximate Riemann solvers, the algorithm (18)-(19) can be interpreted as the well-balanced scheme derived by Greenberg and LeRoux [15] or by Gosse and LeRoux [11], for which the 
condition (15)-(17) is verified.

The quasi-steady wave-propagation algorithm The basic idea of the method developed by LeVêque [27] is to introduce a new Riemann problem in the center of each mesh cell, with values $u_{i}^{-}$on the left half of the cell and $u_{i}^{+}$on the right half, whose flux difference exactly cancels the effect of the source term. These artificial states are defined so that the cell-average is preserved,

$$
u_{i}^{-}=u_{i}-\delta_{i}, \quad u_{i}^{+}=u_{i}+\delta_{i}, \quad \frac{1}{2}\left(u_{i}^{-}+u_{i}^{+}\right)=u_{i}
$$

moreover, if $\delta_{i}$ is chosen according to the in-cell balance condition

$$
A\left(u_{i}^{+}\right)-A\left(u_{i}^{-}\right)+z_{i}^{\prime} b\left(u_{i}\right) \Delta x_{i}=0,
$$

then the jumps occurring at the cell interfaces will correspond to perturbations from the steady states. Note that (23) represents a discrete version of the stationary problem associated with equation (1). The explicit formula for the scheme thus obtained looks like the classical Godunov solver,

$$
\Delta x_{i} \frac{d u_{i}}{d t}+\left(\Delta^{+} A\left(u_{i-1}^{+}, u_{i}^{-}\right)+\Delta^{-} A\left(u_{i}^{+}, u_{i+1}^{-}\right)\right)=0,
$$

with

$$
\begin{aligned}
& \Delta^{+} A\left(u_{i-1}^{+}, u_{i}^{-}\right)=A\left(u_{i}^{-}\right)-A\left(u_{i-\frac{1}{2}}^{*}\right) \\
& \Delta^{-} A\left(u_{i}^{+}, u_{i+1}^{-}\right)=A\left(u_{i+\frac{1}{2}}^{*}\right)-A\left(u_{i}^{+}\right)
\end{aligned}
$$

where $u_{i+\frac{1}{2}}^{*}$ now denotes the solution to the modified Riemann problem at the cell interfaces, between values $u_{i}^{+}$and $u_{i+1}^{-}$. If the solution we are looking for is quasi-steady then we deduce from (22) and (23) that $u_{i}^{+} \approx u_{i+1}^{-}$, as $\delta_{i}$ tends to 0 , so that the steady states are asymptotically preserved.

As for previous examples, this method can extend to any consistent numerical flux function $\mathcal{A}$, by rewriting the flux differences (24)-(25) in the more general form

$$
\begin{aligned}
& \Delta^{+} A\left(u_{i-1}^{+}, u_{i}^{-}\right)=A\left(u_{i}^{-}\right)-\mathcal{A}\left(u_{i-1}^{+}, u_{i}^{-}\right), \\
& \Delta^{-} A\left(u_{i}^{+}, u_{i+1}^{-}\right)=\mathcal{A}\left(u_{i}^{+}, u_{i+1}^{-}\right)-A\left(u_{i}^{+}\right) .
\end{aligned}
$$

Jin's formulas A simple scheme for handling hyperbolic systems of conservation laws with source terms is proposed in [17], which preserves the steady state solutions exactly at the cell interfaces. For methods based on generalized Riemann solvers, it takes form (7) and the source term is discretized by

$$
\mathcal{B}_{i-\frac{1}{2}}^{+}+\mathcal{B}_{i+\frac{1}{2}}^{-}=\frac{A_{i+\frac{1}{2}}-A_{i-\frac{1}{2}}}{D_{i+\frac{1}{2}}-D_{i-\frac{1}{2}}}\left(z_{i+\frac{1}{2}}-z_{i-\frac{1}{2}}\right),
$$


using interface values $A_{i+\frac{1}{2}}=A\left(u_{i+\frac{1}{2}}\right)$ and $D_{i+\frac{1}{2}}=D\left(u_{i+\frac{1}{2}}\right)$, rather than the cell-averages. Consequently, if one gets a steady state at the interfaces,

$$
D_{i+\frac{1}{2}}+z_{i+\frac{1}{2}}=C^{s t}, \quad \forall i \in \mathbb{Z},
$$

a direct computation leads to verify that it is preserved, as we have

$$
\left(A_{i+\frac{1}{2}}-A_{i-\frac{1}{2}}\right)+\frac{A_{i+\frac{1}{2}}-A_{i-\frac{1}{2}}}{D_{i+\frac{1}{2}}-D_{i-\frac{1}{2}}}\left(z_{i+\frac{1}{2}}-z_{i-\frac{1}{2}}\right)=0 .
$$

A more generic scheme, again proposed in [17], is defined by

$$
\Delta x_{i} \frac{d u_{i}}{d t}+\left(A_{i+\frac{1}{2}}-A_{i-\frac{1}{2}}\right)+\frac{b_{i-\frac{1}{2}}+b_{i+\frac{1}{2}}}{2}\left(z_{i+\frac{1}{2}}-z_{i-\frac{1}{2}}\right)=0 .
$$

Although it is not possible to derive an explicit expression of $D$ for a general flux function $A$, some applications considered by the author (shallow water equations, for instance) show this method yields formally second order approximations to the steady states at the interfaces, as suggested by an asymptotic expansion of (27).

The numerical discretizations formulated by Jin are called Steady State Capturing schemes, that is a weaker definition since only the interface values are preserved. According to the idea to process the source term by making use explicitly of relations on the steady states, a Steady State Preserving variation of (26), which agrees with the general formalism (7)-(10), is given by

$$
\begin{aligned}
\mathcal{B}_{i-\frac{1}{2}}^{+} & =\frac{\mathcal{A}\left(u_{i-1}, u_{i}\right)-A\left(u_{i}\right)}{D\left(u_{i-1}\right)-D\left(u_{i}\right)}\left(z_{i}-z_{i-1}\right), \\
\mathcal{B}_{i+\frac{1}{2}}^{-} & =\frac{\mathcal{A}\left(u_{i}, u_{i+1}\right)-A\left(u_{i}\right)}{D\left(u_{i+1}\right)-D\left(u_{i}\right)}\left(z_{i+1}-z_{i}\right) .
\end{aligned}
$$

Again we can readily check the condition (15)-(17) for this method.

\section{Consistency}

In order to investigate theoretical properties of the Upwind Interface Source method, a crucial question to discuss is that equation (7) verifies the consistency with the continuous equation (1)-(4).

We indicate a rigorous definition of consistency, which also results to be satisfied by well-balanced schemes.

Definition 1. A numerical scheme in form (7)-(11) is said to be consistent with (1) if the following limit is verified, locally uniformly in u,

$$
\lim _{\lambda \rightarrow 0} \frac{\mathcal{B}^{+}(u, u, \lambda)+\mathcal{B}^{-}(u, u, \lambda)}{\lambda}=b(u) .
$$


We point out that the above definition of consistency for the source term, in finite volume sense, does not imply that the consistency error vanishes just as for the flux terms. Indeed, because of the choice of an arbitrary nonuniform spatial mesh, the space-step $\Delta x_{i}$ could be very different from the length of an interfacial interval $\Delta x_{i+\frac{1}{2}}=\left|x_{i+1}-x_{i}\right|=\Delta x_{i} / 2+\Delta x_{i+1} / 2$; therefore, the corresponding interfacial discretizations (12) and (13) may have very different values. The condition (28) we have established is closer to (13), which is the most appropriate interpretation of the discrete source term for the general method illustrated in this paper.

As it will be seen clearly in next section, consistency plays an important role to achieve convergence properties of a numerical solver, in particular to prove that the strong limit of discrete approximations (as the mesh is refined) is the suitable weak solution of the continuous problem.

The following result guarantees consistency for the numerical schemes described in Section 3.

Lemma 2. We assume $D$ is monotonic. Let a numerical solver for the system (1)-(4) satisfy the conditions (15)-(17) in Lemma 1, then the property (28) is verified. In other words, all Steady State Preserving schemes are consistent.

Proof. We perform a Taylor expansion of the relation (17) and we deduce that, for some $\xi \in(u, v)$,

$$
D^{\prime}(\xi)(u-v)=z_{+}-z_{-} .
$$

After adding equality (15) to (16), thanks to the definition (3) and (6), this leads to

$$
\begin{aligned}
\mathcal{B}^{+}\left(u, v, z_{+}-z_{-}\right)+\mathcal{B}^{-}\left(u, v, z_{+}-z_{-}\right) & =A(u)-A(v) \\
& =\frac{a(\zeta)}{D^{\prime}(\xi)}\left(z_{+}-z_{-}\right)
\end{aligned}
$$

for some $\zeta \in(u, v)$. We also note that the regularity assumed for the numerical functions enables to perform the general approximations

$$
\begin{aligned}
\mathcal{B}^{ \pm}\left(u, v, z_{+}-z_{-}\right) & =\mathcal{B}^{ \pm}\left(u, u, z_{+}-z_{-}\right) \\
& +\frac{\partial}{\partial v} \mathcal{B}^{ \pm}\left(u, u, z_{+}-z_{-}\right)(v-u)+O\left(|v-u|^{2}\right) .
\end{aligned}
$$

It thus follows from (11) that

$$
\frac{\partial}{\partial v} \mathcal{B}^{+}(u, u, 0)=\frac{\partial}{\partial v} \mathcal{B}^{-}(u, u, 0)=0
$$

and, in view of (29), we obtain that

$$
\begin{aligned}
& \lim _{z_{+}-z_{-} \rightarrow 0} \frac{\mathcal{B}^{+}\left(u, u, z_{+}-z_{-}\right)+\mathcal{B}^{-}\left(u, u, z_{+}-z_{-}\right)}{z_{+}-z_{-}} \\
& =\lim _{z_{+}-z_{-} \rightarrow 0} \frac{\mathcal{B}^{+}\left(u, v, z_{+}-z_{-}\right)+\mathcal{B}^{-}\left(u, v, z_{+}-z_{-}\right)}{z_{+}-z_{-}} .
\end{aligned}
$$


By combining relation (30) with (31), since

$$
\frac{a(\zeta)}{D^{\prime}(\xi)} \longrightarrow \frac{a(u)}{D^{\prime}(u)}=b(u),
$$

we finally conclude that the property (28) is satisfied.

\section{A Lax-Wendroff type convergence theorem}

We are now interested in the convergence of the numerical scheme (7), as the mesh size tends to zero, by analyzing the convergence properties of its solution $\left\{u_{i}(t)\right\}_{i \in \mathbb{Z}}$.

A discretization of the initial condition is given, for instance, by the sequence

$$
u_{i}^{0}=\frac{1}{\left|\mathcal{C}_{i}\right|} \int_{\mathcal{C}_{i}} u^{0}(x) d x, \quad i \in \mathbb{Z} .
$$

As a measure of mesh refinement, we consider the parameter

$$
h=\sup _{i \in \mathbb{Z}} \Delta x_{i} .
$$

For our purpose, we introduce the piecewise constant function $u_{h}$, defined a.e. in $\mathbb{R}_{+} \times \mathbb{R}$ by

$$
u_{h}(t, x)=\sum_{i \in \mathbb{Z}} u_{i}(t) \mathbb{I}_{\mathcal{C}_{i}}(x),
$$

and we study the convergence towards a solution to the problem (1)-(2), as $h$ tends to 0 .

Theorem 1. Assume $z \in W^{1,1}$ for the source term (4). Let $u_{h}$ be obtained from a numerical scheme in form (7)-(11), which satisfies the consistency condition (28). Suppose there exists a constant $C$ such that, uniformly in $h$,

$$
\left\|u_{h}\right\|_{L_{l o c}^{\infty}\left(\mathbb{R}_{+} \times \mathbb{R}\right)} \leq C
$$

and that $u_{h}$ converges to a function $u$ in $L_{\text {loc }}^{1}\left(\mathbb{R}_{+} \times \mathbb{R}\right)$, as $h$ tends to 0 . Moreover, we assume either that, for all bounded intervals $I$ of $\mathbb{R}$,

$$
\sum_{i \in K} \Delta x_{i+\frac{1}{2}}\left|u_{i+1}(t)-u_{i}(t)\right| \stackrel{h \rightarrow 0}{\longrightarrow} 0, \quad \text { in } L_{l o c}^{1}\left(\mathbb{R}_{+}\right),
$$

where $K$ denotes the set of indices such that $x_{i} \in I$; or a geometrical constraint on the spatial mesh, that is

$$
\exists \alpha, \beta>0 \text { so that } \alpha \Delta x_{i+1} \leq \Delta x_{i} \leq \beta \Delta x_{i+1}, \quad \forall i \in \mathbb{Z} .
$$

Then $u$ is a weak solution to the initial value problem (1)-(2), i.e.

$$
\frac{\partial u}{\partial t}+\frac{\partial A(u)}{\partial x}+z^{\prime}(x) b(u)=0, \quad u(0, x)=u^{0}(x), \quad \text { in } \mathcal{D}^{\prime}\left(\mathbb{R}_{+} \times \mathbb{R}\right) .
$$


Proof. The proof is an adaptation of the classical Lax-Wendroff theorem [25] for homogeneous systems of conservation laws.

Let $\varphi \in \mathcal{C}_{0}^{1}\left(\mathbb{R}_{+} \times \mathbb{R}\right)$ be a test function and set

$$
\varphi_{i}(t)=\varphi\left(t, x_{i}\right), \quad i \in \mathbb{Z} .
$$

After multiplying equation (7) by $\varphi_{i}$, we sum over $i$ and integrate in $d t$, to obtain

$$
\begin{aligned}
\int_{\mathbb{R}_{+}} \sum_{i \in \mathbb{Z}} \Delta x_{i} \frac{d u_{i}}{d t} \varphi_{i} d t & +\int_{\mathbb{R}_{+}} \sum_{i \in \mathbb{Z}}\left(A_{i+\frac{1}{2}}-A_{i-\frac{1}{2}}\right) \varphi_{i} d t \\
& +\int_{\mathbb{R}_{+}} \sum_{i \in \mathbb{Z}}\left(\mathcal{B}_{i-\frac{1}{2}}^{+}+\mathcal{B}_{i+\frac{1}{2}}^{-}\right) \varphi_{i} d t=0 .
\end{aligned}
$$

An integration by parts in the first term and a summation by parts in the other ones give

$$
\begin{aligned}
& \int_{\mathbb{R}_{+}} \sum_{i \in \mathbb{Z}} \Delta x_{i} u_{i} \frac{d \varphi_{i}}{d t} d t+\int_{\mathbb{R}_{+}} \sum_{i \in \mathbb{Z}} A_{i+\frac{1}{2}}\left(\varphi_{i+1}-\varphi_{i}\right) d t \\
& -\int_{\mathbb{R}_{+}} \sum_{i \in \mathbb{Z}}\left(\mathcal{B}_{i+\frac{1}{2}}^{+} \varphi_{i+1}+\mathcal{B}_{i+\frac{1}{2}}^{-} \varphi_{i}\right) d t+\sum_{i \in \mathbb{Z}} \Delta x_{i} u_{i}^{0} \varphi_{i}(0)=0 .
\end{aligned}
$$

We define a.e. in $\mathbb{R}_{+} \times \mathbb{R}$ the piecewise constant functions $A_{h}$ and $\mathcal{B}_{h}$, associated with the numerical flux and source term by

$$
\begin{gathered}
A_{h}(t, x)=\mathcal{A}\left(u_{i}, u_{i+1}\right), \\
\mathcal{B}_{h}(t, x)=\frac{1}{\Delta x_{i+\frac{1}{2}}}\left(\mathcal{B}^{+}\left(u_{i}, u_{i+1}, z_{i+1}-z_{i}\right)+\mathcal{B}^{-}\left(u_{i}, u_{i+1}, z_{i+1}-z_{i}\right)\right),
\end{gathered}
$$

for $t \in \mathbb{R}_{+}$and $x \in\left[x_{i}, x_{i+1}\right)$, recalling that $\Delta x_{i+\frac{1}{2}}=\left|x_{i+1}-x_{i}\right|=\frac{\Delta x_{i}}{2}+\frac{\Delta x_{i+1}}{2}$. Next, according to (36), we introduce the piecewise constant approximation of the test function

$$
\varphi_{h}(t, x)=\varphi_{i}(t), \quad t \in \mathbb{R}_{+}, x \in \mathcal{C}_{i},
$$

which converges to $\varphi$ (together with $\frac{\partial \varphi_{h}}{\partial t}$ towards $\left.\frac{\partial \varphi}{\partial t}\right)$ uniformly in $\mathcal{C}_{0}\left(\mathbb{R}_{+} \times \mathbb{R}\right)$, as $h$ tends to 0 . We also consider a continuous piecewise linear function $\psi_{h}$ such that

$$
\begin{aligned}
\psi_{h}\left(t, x_{i}\right) & =\varphi_{i}(t), \quad i \in \mathbb{Z}, \\
\frac{\partial \psi_{h}}{\partial x}(t, x) & =\frac{\varphi_{i+1}(t)-\varphi_{i}(t)}{\Delta x_{i+\frac{1}{2}}}, \quad t \in \mathbb{R}_{+}, x \in\left[x_{i}, x_{i+1}\right),
\end{aligned}
$$

so that $\psi_{h}$ and $\frac{\partial \psi_{h}}{\partial x}$ converge respectively to $\varphi$ and $\frac{\partial \varphi}{\partial x}$ in $\mathcal{C}_{0}^{1}\left(\mathbb{R}_{+} \times \mathbb{R}\right)$, as $h$ tends to 0 . As a direct consequence of these definitions, we have 


$$
\begin{aligned}
\varphi_{j} & =\frac{\varphi_{i}+\varphi_{i+1}}{2}+O(h), \quad j=i, i+1, \\
\int_{x_{i}}^{x_{i+1}} \psi_{h}(t, x) d x & =\frac{\varphi_{i}+\varphi_{i+1}}{2} \Delta x_{i+\frac{1}{2}} .
\end{aligned}
$$

Taking into account all the relations stated above, we can put the discrete sum (37) into the integral form

$$
\begin{aligned}
& \int_{\mathbb{R}_{+}} \int_{\mathbb{R}} u_{h}(t, x) \frac{\partial \varphi_{h}}{\partial t}(t, x) d x d t+\int_{\mathbb{R}_{+}} \int_{\mathbb{R}} A_{h}(t, x) \frac{\partial \psi_{h}}{\partial x}(t, x) d x d t \\
& -\int_{\mathbb{R}_{+}} \int_{\mathbb{R}} \mathcal{B}_{h}(t, x)\left(\psi_{h}(t, x)+O(h)\right) d x d t+\int_{\mathbb{R}^{\prime}} u_{h}^{0}(x) \varphi_{h}(0, x) d x=0 .
\end{aligned}
$$

As $h$ tends to 0 , passing to the limit in (40), we claim that it turns out

$$
\begin{aligned}
& \int_{\mathbb{R}_{+}} \int_{\mathbb{R}} {\left[u(t, x) \frac{\partial \varphi}{\partial t}(t, x)+A(t, x) \frac{\partial \varphi}{\partial x}(t, x)-B(t, x) \varphi(t, x)\right] d x d t } \\
&+\int_{\mathbb{R}} u^{0}(x) \varphi(0, x) d x=0 .
\end{aligned}
$$

The computation is obvious for the first and the last terms, by exploiting the convergence properties of approximations $u_{h}$ and $\varphi_{h}$.

For the other integrals of (40), the process is less straightforward: as we remarked in Section 4, due to the presence of a variable space-step, the interfacial interval $\left[x_{i}, x_{i+1}\right.$ ) could be really different from the mesh cell $\mathcal{C}_{i}$ (where the conservative unknowns are discretized); so, standard techniques do not work in this case and proving convergence requires the additional hypotheses on the structures that we have imposed.

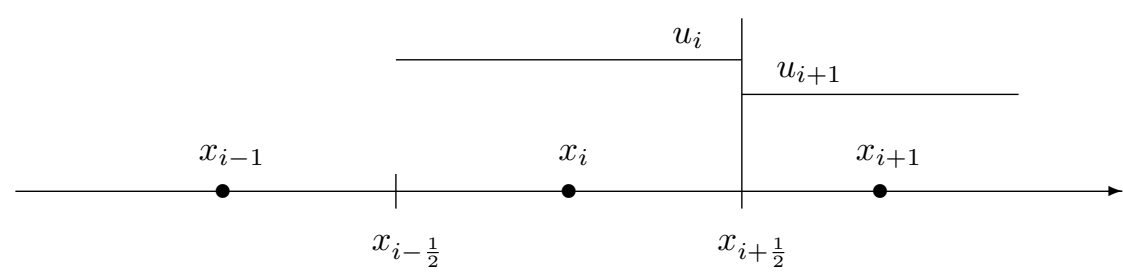

We need to characterize the functions $A(t, x)$ and $B(t, x)$ in equation (41) as the weak limits of $A_{h}(t, x)$ and $\mathcal{B}_{h}(t, x)$, for reproducing them in terms of the numerical unknowns.

We first observe that, thanks to (33) and the properties (8)-(9) of the numerical flux, $A_{h}$ is locally bounded on $\mathbb{R}_{+} \times \mathbb{R}$ (uniformly in $h$ ). Coming back to discrete notation (38), we decompose on the subintervals $\left[x_{i}, x_{i+\frac{1}{2}}\right)$ and $\left[x_{i+\frac{1}{2}}, x_{i+1}\right)$, rearranging terms as follows, 


$$
\begin{aligned}
A_{h}(t, x) & =\sum_{i \in \mathbb{Z}} A\left(u_{i}\right) \mathbb{I}_{\left[x_{i-\frac{1}{2}}, x_{i+\frac{1}{2}}\right)}(x) \\
& +\sum_{i \in \mathbb{Z}}\left[\mathcal{A}\left(u_{i}, u_{i+1}\right)-A\left(u_{i}\right)\right] \mathbb{I}_{\left[x_{i}, x_{i+\frac{1}{2}}\right)}(x) \\
& +\sum_{i \in \mathbb{Z}}\left[\mathcal{A}\left(u_{i-1}, u_{i}\right)-A\left(u_{i}\right)\right] \mathbb{I}_{\left[x_{i-\frac{1}{2}}, x_{i}\right)}(x) \\
& =A\left(u_{h}(t, x)\right)+\mathcal{R}_{h}^{+}(t, x)+\mathcal{R}_{h}^{-}(t, x) .
\end{aligned}
$$

We conclude that $A(u(t, x))$ is the expected value for the limit $A(t, x)$, as $h$ tends to 0 , provided that the two remainders in (42) vanish. We only treat with $\mathcal{R}_{h}^{+}$, the other one results in the same way. According to definition (32), since $u_{h}$ converges to a function $u$ in $L_{\text {loc }}^{1}\left(\mathbb{R}_{+} \times \mathbb{R}\right)$, we also derive

$$
\sum_{i \in \mathbb{Z}}\left|u_{i}(t)-\bar{u}_{i}(t)\right| \mathbb{I}_{\mathcal{C}_{i}}(x) \underset{L_{l o c}^{1}}{\longrightarrow} \underset{\mathbb{R}+\times \mathbb{R})}{\longrightarrow} 0
$$

where the sequence $\left\{\bar{u}_{i}(t)\right\}_{i \in \mathbb{Z}}$ is defined by the cell-averages of $u$ on the discretization mesh. Let $I$ be any bounded interval of $\mathbb{R}$ and we denote by $C_{\mathcal{A}}$ a Lipschitz constant for $\mathcal{A}$, then the assumptions on the numerical flux lead to estimate (with the notation set out above in the theorem)

$$
\begin{aligned}
\int_{I}\left|\mathcal{R}_{h}^{+}(t, x)\right| d x & \leq \int_{I} \sum_{i \in \mathbb{Z}}\left|\mathcal{A}\left(u_{i}, u_{i+1}\right)-A\left(u_{i}\right)\right| \mathbb{I}_{\left[x_{i}, x_{i+\frac{1}{2}}\right)}(x) d x \\
& \leq C_{\mathcal{A}} \sum_{i \in K} \frac{\Delta x_{i}}{2}\left|u_{i+1}-u_{i}\right| .
\end{aligned}
$$

Under the hypothesis (34), this last term vanishes and the conclusion is done. Otherwise, we can further manipulate the previous bound by introducing appropriate quantities, that is

$$
\begin{aligned}
\sum_{i \in K} \Delta x_{i}\left|u_{i+1}-u_{i}\right| & \leq \sum_{i \in K} \Delta x_{i}\left|u_{i+1}-\bar{u}_{i+1}\right| \\
& +\sum_{i \in K} \Delta x_{i}\left|\bar{u}_{i+1}-\bar{u}_{i}\right|+\sum_{i \in K} \Delta x_{i}\left|\bar{u}_{i}-u_{i}\right| .
\end{aligned}
$$

In particular, for the alternative hypothesis of nondegeneracy (35) made on the mesh, we have

$$
\sum_{i \in K} \Delta x_{i}\left|u_{i+1}-\bar{u}_{i+1}\right| \leq \beta \sum_{i \in K} \Delta x_{i+1}\left|u_{i+1}-\bar{u}_{i+1}\right|,
$$

so that property (43) ensures that first and last sum in the right-hand side of (45) vanish, as $h$ tends to 0 . It remains the second term to be studied, involving only the function $u$, which immediately converges to 0 if $u \in B V$ (or smooth enough); this result also holds for $u \in L^{1}$, by applying standard 
regularization arguments (we define $u^{\epsilon} \in B V, u^{\epsilon} \rightarrow u$ in $L^{1}$, then we perform an estimation on the cell-averages $\bar{u}_{i}$ and $\bar{u}_{i}^{\epsilon}$ like in (45) and we finally conclude by combining convergence properties).

We now pass to the source term, to which a similar procedure applies. Taking into account the definition (39), setting $\mathcal{B}=\mathcal{B}^{+}+\mathcal{B}^{-}$, we can write

$$
\mathcal{B}_{h}(t, x)=\sum_{i \in \mathbb{Z}} \frac{\mathcal{B}\left(u_{i}, u_{i+1}, z_{i+1}-z_{i}\right)}{z_{i+1}-z_{i}} \cdot \frac{z_{i+1}-z_{i}}{\Delta x_{i+\frac{1}{2}}} \mathbb{I}_{\left[x_{i}, x_{i+1}\right)}(x) .
$$

Notice that the hypothesis $z \in W^{1,1}$ guarantees that discrete differences converge to the derivative $z^{\prime} \in L^{1}$; together with condition (28), this leads to justify the assertion that $\mathcal{B}_{h}(t, x)$ is $L^{1}-$ weak bounded in $\mathbb{R}_{+} \times \mathbb{R}$. We proceed as in (42), by performing the following decomposition

$$
\begin{aligned}
\mathcal{B}_{h}(t, x) & =\sum_{i \in \mathbb{Z}} \frac{\mathcal{B}\left(u_{i}, u_{i}, z_{i+1}-z_{i}\right)}{z_{i+1}-z_{i}} \cdot \frac{z_{i+1}-z_{i}}{\Delta x_{i+\frac{1}{2}}} \mathbb{I}_{\left[x_{i}, x_{i+\frac{1}{2}}\right)}(x)+\mathcal{E}_{h}^{+}(t, x) \\
& +\sum_{i \in \mathbb{Z}} \frac{\mathcal{B}\left(u_{i}, u_{i}, z_{i}-z_{i-1}\right)}{z_{i}-z_{i-1}} \cdot \frac{z_{i}-z_{i-1}}{\Delta x_{i-\frac{1}{2}}} \mathbb{I}_{\left[x_{i-\frac{1}{2}}, x_{i}\right)}(x)+\mathcal{E}_{h}^{-}(t, x) .
\end{aligned}
$$

The sum of the two principal terms of (46) converges to $z^{\prime}(x) b(u)$, in view of the arguments just mentioned and the strong convergence of $u_{h}$ towards $u$, by means of Lebesgue's theorem. For the remainders, we give details in the case of $\mathcal{E}_{h}^{+}(t, x)$, for instance. We then have

$$
\begin{aligned}
\mathcal{E}_{h}^{+}(t, x)=\sum_{i \in \mathbb{Z}} \frac{1}{\Delta x_{i+\frac{1}{2}}} & \left(\mathcal{B}\left(u_{i}, u_{i+1}, z_{i+1}-z_{i}\right)\right. \\
& \left.-\mathcal{B}\left(u_{i}, u_{i}, z_{i+1}-z_{i}\right)\right) \mathbb{I}_{\left[x_{i}, x_{i+\frac{1}{2}}\right)}(x) .
\end{aligned}
$$

The analogue of estimation (44) for (47) becomes

$$
\begin{aligned}
\int_{I}\left|\mathcal{E}_{h}^{+}(t, x)\right| d x & \leq \sum_{i \in K}\left|\mathcal{B}\left(u_{i}, u_{i+1}, z_{i+1}-z_{i}\right)-\mathcal{B}\left(u_{i}, u_{i}, z_{i+1}-z_{i}\right)\right| \\
& =\sum_{i \in K}\left|\int_{u_{i}}^{u_{i+1}} \frac{\partial \mathcal{B}}{\partial v}\left(u_{i}, v, z_{i+1}-z_{i}\right) d v\right| \\
& =\sum_{i \in K}\left|\int_{u_{i}}^{u_{i+1}}\left(\frac{\partial \mathcal{B}}{\partial v}\left(u_{i}, v, z_{i+1}-z_{i}\right)-\frac{\partial \mathcal{B}}{\partial v}\left(u_{i}, v, 0\right)\right) d v\right| \\
& \leq C_{\mathcal{B}} \sum_{i \in K}\left|u_{i+1}-u_{i}\right|\left|z_{i+1}-z_{i}\right|,
\end{aligned}
$$

where we exploited property (11) and the regularity assumed in (10). Then we can regularize the function $z$ by introducing $z^{\epsilon} \in W^{1, \infty}, z^{\epsilon} \rightarrow z$ in $W^{1,1}$, for which we write 


$$
\begin{aligned}
\sum_{i \in K}\left|u_{i+1}-u_{i}\right|\left|z_{i+1}-z_{i}\right| & \leq \sum_{i \in K}\left|u_{i+1}-u_{i}\right|\left|z_{i+1}-z_{i+1}^{\epsilon}\right| \\
& +\sum_{i \in K}\left|u_{i+1}-u_{i}\right|\left|z_{i}^{\epsilon}-z_{i}\right|+\sum_{i \in K}\left|u_{i+1}-u_{i}\right|\left|z_{i+1}^{\epsilon}-z_{i}^{\epsilon}\right| \\
& \leq 4 C \sum_{i \in K}\left|z_{i}^{\epsilon}-z_{i}\right|+C_{\epsilon} \sum_{i \in K} \Delta x_{i+\frac{1}{2}}\left|u_{i+1}-u_{i}\right|,
\end{aligned}
$$

with $C$ defined as in (33) and $C_{\epsilon}$ only depending on the regularization. The same procedure we have considered before allow us to conclude that the upper bound (48) vanishes, as $h$ tends to 0 .

Thanks to the previous computations, we have proved that the limit function $u$ satisfies the weak formulation of the Cauchy problem (1)-(2),

$$
\begin{aligned}
\int_{\mathbb{R}_{+}} \int_{\mathbb{R}}\left[u(t, x) \frac{\partial \varphi}{\partial t}(t, x)+\right. & \left.A(u(t, x)) \frac{\partial \varphi}{\partial x}(t, x)-z^{\prime}(x) b(u(t, x)) \varphi(t, x)\right] d x d t+ \\
& +\int_{\mathbb{R}} u^{0}(x) \varphi(0, x) d x=0,
\end{aligned}
$$

so that the proof of the theorem is completed.

Remark. For the particular case of uniform spatial mesh, i.e. $\Delta x_{i}=h$, $\forall i \in \mathbb{Z}$, sets of weaker hypotheses can be considered and the proof is simplified, due to the fact that the consistency error vanishes for the source term (refer to [9] and [26]). The version presented above is compatible with those general statements (see also [10]) and actually extends the classical Lax-Wendroff theorem to scalar conservation laws with a source term.

\section{Conclusion and remarks}

We have proposed a consistency condition for hyperbolic conservation laws with a source term $z^{\prime}(x) b(u)$, discretized according to the Upwind Interface Source method. We have proved that numerical schemes which preserve the steady state solutions are consistent in that sense. Moreover, a strong limit of discrete solutions satisfies the continuous equation, as the mesh is refined. Theorem 1 thus constitutes a fundamental result in the theoretical analysis of the numerical method.

Nevertheless, the conditions established in the previous sections do not guarantee that discrete approximations given by (7)-(11) do converge and we do not know whether the limit weak solution is the unique physical solution of the Cauchy problem (1)-(4). For that we need to precise some criteria of stability for the approximate solution and we have to consider further assumptions on the discrete functions to derive suitable error estimates. 
In order to ensure that a weak solution obtained as limit of (32) satisfies the family of entropy inequalities (5), it suffices to show that a discrete entropy inequality,

$$
\Delta x_{i} \frac{d}{d t} S\left(u_{i}\right)+\left(\eta_{i+\frac{1}{2}}^{S}-\eta_{i-\frac{1}{2}}^{S}\right)+\mathcal{B}_{i-\frac{1}{2}}^{S,+}+\mathcal{B}_{i+\frac{1}{2}}^{S,-} \leq 0
$$

holds for the numerical scheme, with the usual formalism

$$
\eta_{i+\frac{1}{2}}^{S}=\eta^{S}\left(u_{i}, u_{i+1}\right), \quad \mathcal{B}_{i+\frac{1}{2}}^{S, \pm}=\mathcal{B}^{S, \pm}\left(u_{i}, u_{i+1}, z_{i+1}-z_{i}\right)
$$

where $\eta^{S}$ and $\mathcal{B}^{S, \pm}$ are some numerical entropy flux function and source term, which must be consistent with $\eta(u)$ and $S^{\prime}(u) B(x, u)$ in the same way that we required $\mathcal{A}$ and $\mathcal{B}^{ \pm}$to be consistent with $A(u)$ and $B(x, u)$ in (1). Therefore, mimicking the proof of the Lax-Wendroff theorem, we can prove that the weak form of the entropy inequality (5) is also verified.

Note that the possibility to write formulas (49)-(50) relies only on the characterization of the numerical flux (namely, at least the condition of $E$-scheme has to be assumed); for the source term, the definition is automatically made, since we have

$$
\mathcal{B}_{i-\frac{1}{2}}^{S,+}=S^{\prime}\left(u_{i}\right) \mathcal{B}_{i-\frac{1}{2}}^{+}, \quad \mathcal{B}_{i+\frac{1}{2}}^{S,-}=S^{\prime}\left(u_{i}\right) \mathcal{B}_{i+\frac{1}{2}}^{-}
$$

The question to determine the order of accuracy of the numerical scheme by means of error estimates is more delicate than for the homogeneous system, due to the presence of source terms. The approach formulated by Kruzkov is used henceforth in the literature (see [11],[12] and [19],[20] for instance), providing a method to convert any discrete entropy inequality into an error estimate. The general procedure consists in the following formulation for the approximate solution, in $\mathcal{D}^{\prime}\left(\mathbb{R}_{+} \times \mathbb{R}\right)$,

$$
\frac{\partial S\left(u_{h}\right)}{\partial t}+\frac{\partial}{\partial x} \eta^{S}\left(u_{h}\right)+S^{\prime}\left(u_{h}\right) z^{\prime}(x) b\left(u_{h}\right) \leq \frac{\partial}{\partial x} \operatorname{Err}_{2}(t, x)+\operatorname{Err}_{1}(t, x),
$$

where we set

$$
\begin{aligned}
& \frac{\partial}{\partial x} \operatorname{Err}_{1}(t, x)=\frac{\partial}{\partial x} \eta^{S}\left(u_{h}\right)-\sum_{i \in \mathbb{Z}} \frac{1}{\Delta x_{i}}\left(\eta_{i+\frac{1}{2}}^{S}-\eta_{i-\frac{1}{2}}^{S}\right) \mathbb{I}_{\mathcal{C}_{i}}(x) \\
& \operatorname{Err}_{2}(t, x)=S^{\prime}\left(u_{h}\right) z^{\prime}(x) b\left(u_{h}\right)-\sum_{i \in \mathbb{Z}} \frac{1}{\Delta x_{i}}\left(\mathcal{B}_{i-\frac{1}{2}}^{S,+}+\mathcal{B}_{i+\frac{1}{2}}^{S,-}\right) \mathbb{I}_{\mathcal{C}_{i}}(x)
\end{aligned}
$$

Then the results of [3] apply to this particular problem, to deduce stability bounds and conclude the convergence properties we have assumed in Theorem 1. We remark that regularity hypotheses like (34) and (35) are necessary to control the variations of the numerical solution in comparison with the space-step (refer to [30] for the homogeneous problem). 
In order to avoid BV estimates, which are not available in case of insufficiently smooth source terms and for multidimensional systems on an unstructured mesh, arguments based on the measure-valued method and the so-called weak BV estimates are developed (see [7] and [4],[5], for instance) or the kinetic approach presented in [2].

\section{References}

1. Bermudez A., Vasquez M.E., Upwind methods for hyperbolic conservation laws with source terms, Comput. ES Fluids, 23 (1994), no. 8, 1049-1071

2. Botchorishvili R., Perthame B., Vasseur A., Equilibrium Schemes for Scalar Conservation Laws with Stiff Sources, Math. Comp., to appear

3. Bouchut F., Perthame B., Kružkov's estimates for scalar conservation laws revisited, Trans. Amer. Math. Soc., 350 (1998), no. 7, 2847-2870

4. Cockburn B., Coquel F., LeFloch P., An error estimate for finite volume methods for multidimensional conservation laws, Math. of Comp., 63 (1994), 77-103

5. Cockburn B., Coquel F., LeFloch P., Convergence of the finite volume method for multidimensional conservation laws, SIAM J. Numer. Anal., 32 (1995), no. 3, 687-705

6. Eymard R., Gallouët T., Herbin R., Finite Volume Methods, Handbook of numerical analysis, vol. VIII, P.G.Ciarlet and J.L.Lions editors, Amsterdam, North-Holland, 2000

7. Eymard R., Gallouët T., Ghilani M., Herbin R., Error estimates for the approximate solutions of a nonlinear hyperbolic equation given by some finite volume schemes, I.M.A. Journal of Numer. Anal., 18 (1998), 563-594

8. Gallouët T., Hérard J.M., Seguin N., Some approximate Godunov schemes to compute shallow-water equations with topography, AIAA-2001 (2000)

9. Godlewski E., Raviart P.A., Hyperbolic systems of conservation laws, Mathématiques \& Applications, n. 3/4, Paris, Ellipses, 1991

10. Godlewski E., Raviart P.A., Numerical approximation of hyperbolic systems of conservation laws, Applied Mathematical Sciences 118, New York, SpringerVerlag, 1996

11. Gosse L., LeRoux A.Y., A well-balanced scheme designed for inhomogeneous scalar conservation laws, C.R. Acad. Sci. Paris Sér.I Math., 323 (1996), no. 5, 543-546

12. Gosse L., A priori error estimate for a well-balanced scheme designed for inhomogeneous scalar conservation laws, C.R. Acad. Sci. Paris Sér.I Math., 327 (1998), no. 5, 467-472

13. Gosse L., A well-balanced flux-vector splitting scheme designed for hyperbolic systems of conservation laws with source terms, Comput. Math. Appl., 39 (2000), no. 9-10, 135-159

14. Gosse L., A well-balanced scheme using non-conservative products designed for hyperbolic systems of conservation laws with source terms, Math. Models Methods Appl. Sci., 11 (2001), no. 2, 339-365

15. Greenberg J.M., LeRoux A.Y., A well balanced scheme for the numerical processing of source terms in hyperbolic equations, SIAM J. Numer. Anal., 33 (1996), 1-16 
16. Greenberg J.M., LeRoux A.Y., Baraille R., Noussair A., Analysis and approximation of conservation laws with source term, SIAM J. Numer. Anal., 34 (1997), no. 5, 1980-2007

17. Jin S., A steady-state capturing method for hyperbolic systems with geometrical source terms, M2AN Math. Model. Numer. Anal., 35 (2001), no. 4, 631-645

18. Karni S., personal communications

19. Katsaounis T., Makridakis C., Finite volume relaxation schemes for multidimensional conservation laws, Math. Comp., 70 (2001), n. 234, 533-553

20. Katsoulakis M.A., Kossioris G., Makridakis C., Convergence and error estimates of relaxation schemes for multidimensional conservation laws, Comm. Partial Differential Equations, 24(1999), n. 3-4, 395-424

21. Kružkov S.N., First order quasilinear equations in several independent space variables, Math. USSR Sb., 10 (1970), 217-243

22. Kurganov A., Central-upwind schemes for balance laws. Application to the Broadwell model, Proceedings of the Third International Symposium on Finite Volumes for Complex Applications (2002), to appear

23. Kurganov A., Levy D., Central-Upwind Schemes for the Saint-Venant system, M2AN Math. Model. Numer. Anal. (2001), to appear

24. Lax P.D., Shock waves and entropy, in Contributions to nonlinear functional analysis, E.H.Zarantonello editor, New York, Academic Press, 1971, 603-634

25. Lax P.D., Wendroff B., Systems of conservations laws, Comm. Pure Appl. Math., 13 (1960), 217-237

26. LeVêque R.J., Numerical Methods for Conservation Laws, Lectures in Mathematics, ETH Zurich, Birkhauser, 1992

27. LeVêque R.J., Balancing source terms and flux gradients in high-resolution Godunov methods: the quasi-steady wave-propagation algorithm, J. Comput. Phys., 146 (1998), no. 1, 346-365

28. Perthame B., Simeoni C., A kinetic scheme for the Saint-Venant system with a source term, Calcolo, 38 (2001), no. 4, 201-231

29. Roe P.L., Upwind differencing schemes for hyperbolic conservation laws with source terms, in Nonlinear Hyperbolic Problems, C.Carasso, P.A.Raviart and D.Serre editors, Lecture Notes in Math., vol. 1270, Berlin, Springer-Verlag, 1987, pp. 41-51

30. Sanders R., On Convergence of Monotone Finite Difference Schemes with Variable Spatial Differencing, Math. Comp., 40 (1983), 91-106

31. Vasseur A., Well-posedness of scalar conservation laws with singular sources, in preparation 